\title{
Synthesis of Novel Quinolinecarboxamide Derivatives with Estrogenic Activity
}

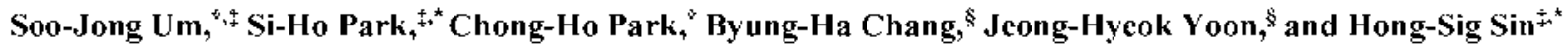 \\ ${ }^{\circ}$ Dept. of Bioscience \& Biotechnologv/Institute of Bioscience, Sejong University, Seonl $143-747$, Korea

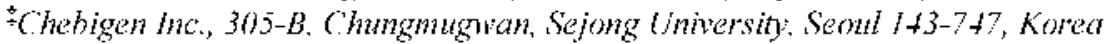

$\$$ IDR Tech, Inc, Research center B-1, Kwacheon Officetel $5^{\text {th }}$ Fl, Byolyang-dong, Kwacheon-shi, Kronggi-do $\left.427-f\right) 40$, Korea Recened January 29, 2003

Key Words : Estrogen, Quinolinecarboxanide derivatives, Virtual screening. Functional group, Fstrogen receptor (F:R)

Even though estrogens have a well-established role in the growth of hormone-dependant tumors by the estrogen receptor (ER)-dependant mitogenic ellect in cells containing $E R,{ }^{\prime}$ a member of the nuclear receptor superfarnily, ${ }^{2}$ they exert numbers of lavorable activities in women. From a therapeutical point of view, estrogen and its derivatives are well known not only as oral contraceptives, but also as a major component in homone replacement therapy (IIRT) required for bone loss prevention and for the control of cardiovascular diseases in postmenopausal women. ${ }^{3}$

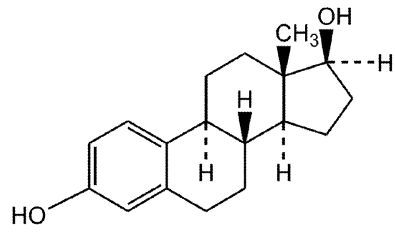

17 -Estradiol (E2)

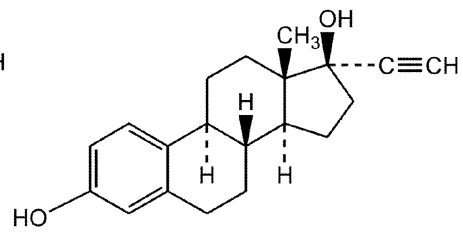

Ethynylestradiol
The most widely used estrogens are $17 \beta$-estradiol ${ }^{4}$ and ethynylestradiol, ${ }^{5}$ a synthetic steroidal compound. These estrogen agonists have been used for postmenopausal women required for IIRT. Ilowever, it has been known that long-term usage of estrogen causes high incidence of breast cancer, which leads to development of antiestrogen such as tamoxifen, the lirst selective ER modulator (SERM). Recently, raloxifene as a sceond SERM was developed to alleviate menopausal symptoms without risk of breast cancer $^{8}$ Alterwards, the more ellective SERMs are being developed and tested that act as antiestrogens on breast and endometrium while having estrogenic effects on bones, the lipid profile and the central nervous system.

In this point of view to develop antiestrogens, we used virtual screening system based on the structure of ER. From the initial screening, we identified compound 4 that has nonsteroidal quinoline strueture. More compounds were synthesized from the leader compound $\mathbf{4}$ and tested for estrogenic activity using $\mathrm{ER} \alpha$ and $\mathrm{ER} \beta$.

\section{Results and Discussion}

Synthesis of quinolinecarboxamide derivatives. $A$ pplication of such multi-component condensation approach based Doebner reation'2 for the synthesis of a clinically usclul

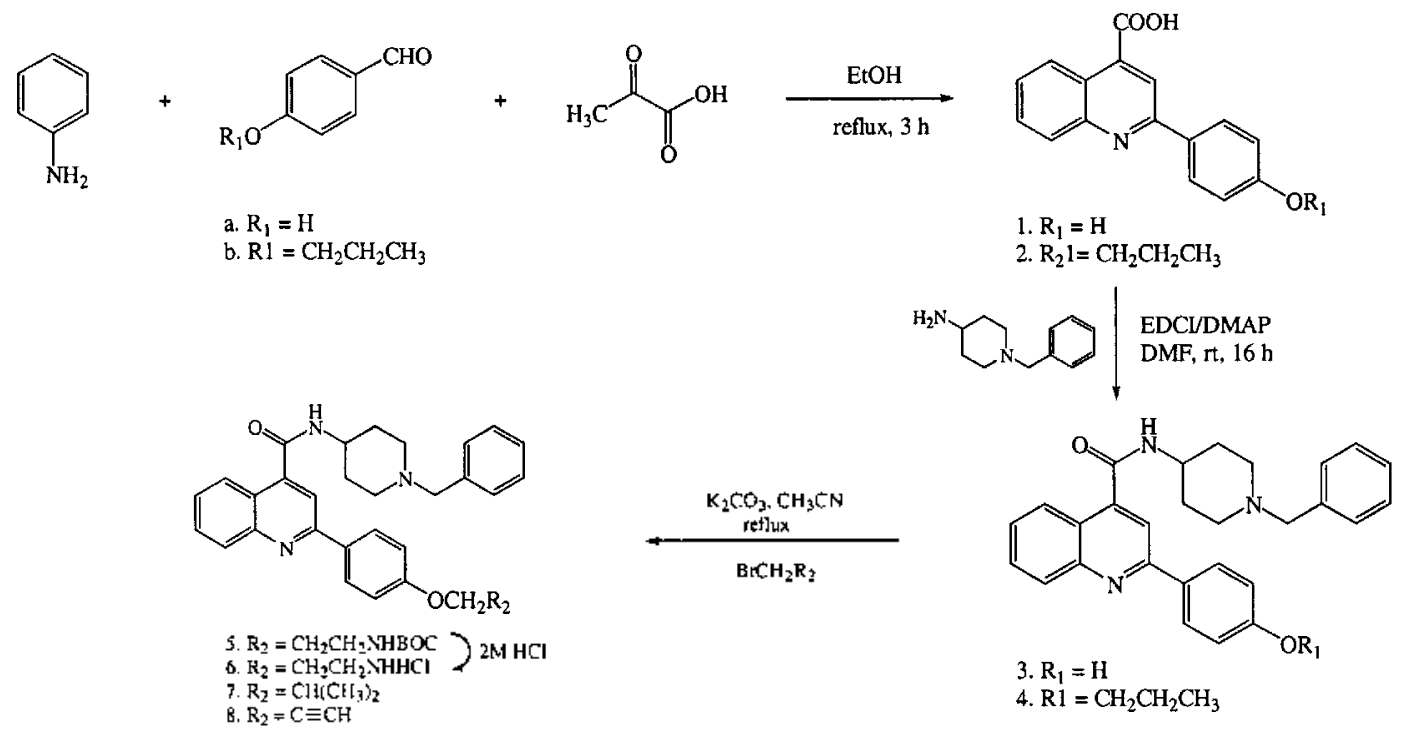

Scheme 1

"Co-corresponding authors. Hong-Sig Sin (Tel: +82-2-465-169l: Fax: +82-2-465-1690; E-mail: shsdora hanmail.net). Si-Ho Park (Tel: 182-2-465-1691: Fax: 182-2-465-1690; t-mail: siho-parkiahanmail.net) 
plarmacophore, 2-(t-hydroxyphenyl)quinolne-t-carboxylic acid 1 is shown in scheme 1 .

The quinolinecarboxylic acid 1 was obtamed using the condensation of anline and pyruxic acid with t-hydrosy benzaldehyde in EtOH under reflux. ${ }^{1.3}$ and coupled with tamino-l-benzylpiperidine using EDCl/DMAP as reagent. ${ }^{14}$ to give quinolinecarboxamide 3 in $76 \%$ yield. Following to the same method. proposy qunolnecarboxamude 4 was obtamed from propoxy quinolinecarboxylic acid 2 in $70 \%$ y eld

The sy nthesis of target compound 6-8 was prepared by $O$ alkylation of the bromo-substitued function groups with quinolinecarboxamide $3 \mathrm{~m}$ the presence of anhydrous potassium carbonate.

1 + -Boc-aminopropoxy qumolinecarboxamide 5 was camied out the $O$-alky lation of quinolinecarboxamide 3 with $\mathrm{N}$-Boc3-bromopropoxyamine using $\mathrm{K}_{2} \mathrm{CO}_{3}$ in refluxing acetonitrile. ${ }^{15}$ followed by the treatment with $2 \mathrm{M} \mathrm{HCl}$ etherate ${ }^{1 / 5}$ generating aminopropoxy quinolinecarboxamide salt 6 in 93 $\%$ overall yield. It should be noted that the solubility of quinolinecarboxamide 3 was transformated from organic solubility to the enhanced aqueous solubility for the plarmacological assay.
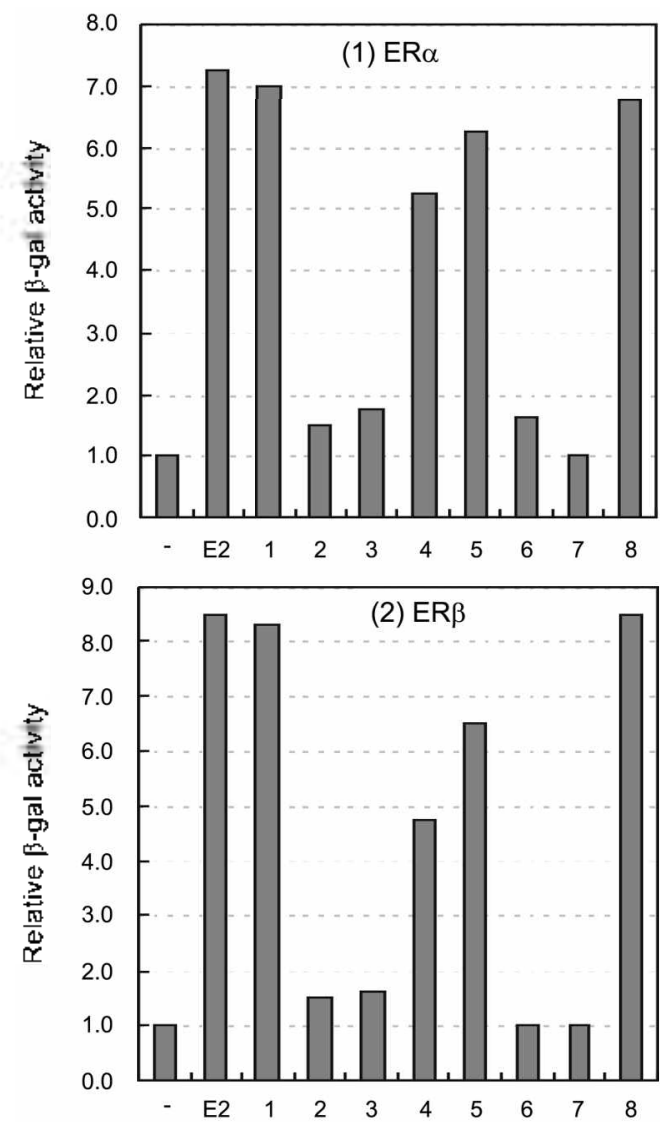

Figure 1. Lstrogenic ellect of sylhetic compounds. NIII3T3 cells wete cotransfected with 3XFRF-tk- $\beta$-gal reporter gente and F.R $\alpha$ (A) or F.R $\beta$ (B) expression vector in pSG5, and treated with $1 \mu \mathrm{M}$ L2 or syllhetic compounds using Lipotectamine PLUS reagent. Lstrogenic eflict was delemined by transeriptional activitics conjled with FR, shown by R-gal activity and exjeressed as a felative activity compared with that of the ] MSSO control (average of 3 independent assays \pm SD).
Aiming to improve the binding activity of 4 . further work was in progress involving structural modifications of quinolinecarboxamide. We expected that the activity of compound 7. 8 would be also affected by the size changes in the quinolinecarboxamide basic side chain.

In order to introduce isobutyl or propy.lnyl group in 4'-OH position. these same conditions applied to 5 gave $77 \%$ and $36 \%$. respectively: of the corresponding quinolinecarboxanude 7.8 to compare their size effect.

Estrogen agonist test. Compound 4 was identified from virtual screening for estrogen antagonist. More derivatives were synthesized using compound 4 as a leader. To determune whether our synthesized compounds act as estrogen agonst or antagonust, we performed transcription assays ${ }^{-}$in which $\mathrm{NlH} 3 \mathrm{~T} 3$ cells were transiently transfected with 3 XERE-tk- $\beta$-gal reporter plasmid. and $\operatorname{ER} \alpha$ or $\operatorname{ER} \beta$ expression vector. As a positive control. $17 \beta$-estradiol $\left(\mathrm{E}_{2}\right)$ was used in DMSO. As expected. $1 \mu \mathrm{M} \mathrm{E}_{2}$ activated the transcriptional activities of both $\mathrm{ER} \alpha$ and $\mathrm{ER} \beta$. Compounds 1. 4. 5. and 8 were active, whereas 2. 3,6, and 7 were
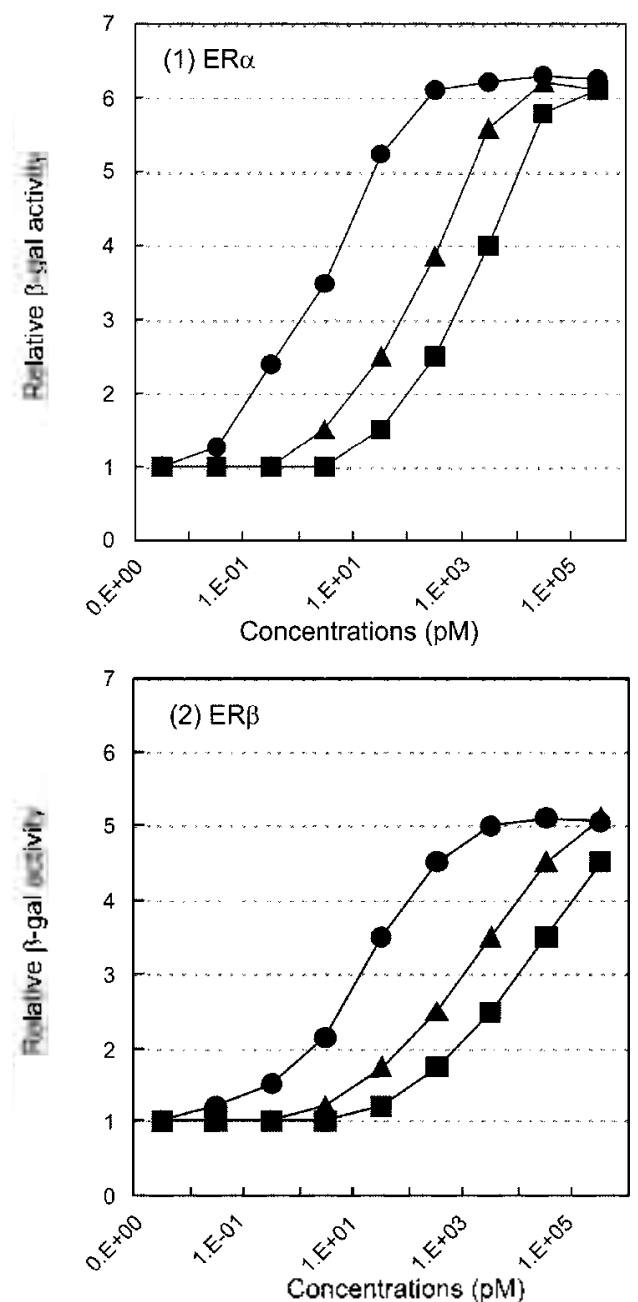

Figure 2. FC. 50 of synthetic compound 1 and 8 . Transtections were conducted as described by increasing concentrations of $17 \beta$ estradiol (L2) ( ) compound 1 ( $\boldsymbol{Q}$ ). or compound 8 ( $\Delta$ ) Irom 0.01 to $100600 \mathrm{pM}$. EC io value. the concentration activating $\mathrm{ER}$ by $50 \%$, was detennined tor F.R $\alpha(A)$ and FRR $(B)$ 
inactive for both $\operatorname{ER} \alpha$ and $\operatorname{ER} \beta$ (Figure l).

Although compound $\downarrow+$ was virtually screened as an antagonist. it acted as an agonist in our assay system. Of active compounds. 1 and $\mathbf{8}$ were selected for further dosedependent assays to determine $\mathrm{EC}_{5 \mathrm{~S}}$ value, the concentration activating ER by $50 \%$ (Figure 2). When compared to $E C_{5 ! 1}$ of $\mathrm{E} 2(0.6 p \mathrm{M}$ for $\mathrm{ER} a$ and $2.5 p \mathrm{M}$ for $\mathrm{ER} \beta)$, those of compound 1 were 500 and $1.000 \mathrm{pM}$ for $\operatorname{ER} \alpha$ and $\operatorname{ER} \beta$. respectively: $E_{S(1)}$ values of compound 8 were 40 and 100 $p \mathrm{M}$ for $\mathrm{ER} \alpha$ and $\mathrm{ER} \beta$. respectively: Therefore, compound 1 is 833.3 and 12.5 times less active than E2 and compound 8 for $\operatorname{ER} \alpha$, respectively. For $E R \beta$. compound 1 is 400 and 10 times less active than E2 and compound 8 . respectively: These data indicate that compound $\mathbf{1}$ and $\mathbf{8}$ are about two times more active to $\mathrm{ER} \alpha$ than ER $\beta$ likewise E2. Furthermore. compound $\mathbf{8}$ is about 10 times more active than compound $\mathbf{1}$ to both ERs. As mentioned, compound 4. a leader for compound $\mathbf{1}$ and $\mathbf{8}$. was designed as an antiestrogen. However. no antagonist effect was found in our assays (data not shown). suggesting that more works should be followed to design antiestrogen.

Throughout our works, we found that non-steroidal quinoline stnicture could be estrogen agonist. These results were unexpected as virtual screening was conducted to identify. estrogen antagonists. Although the reason for the unexpected results is not clarified yet. our screened compound 4 may not possess rigid. bulky. and extended structure in vivo that is required for preventing coactivator from associating with ER for the enhanced transcriptional activity. Therefore. our data may be important and better be considered for virtual screening of real antagonists. Our data also indicate that instead of antagonist. compound $\mathbf{8}$ can be used to design more estrogenic compounds as a second leader.

\section{Experimental Section}

All commercially axailable reagents and solvents were used without further purification. All reactions were conducted under an Ar atmosphere. except for those reactions utilizing water as a solvent. They were monitored by TLC (Merck Kieselgel 60, F254). All the products prepared were purified by flash column chromatography on silica gel 60 (Merck. 230-100 mesh). Melting points were determined with a Büchi 510 hot stage apparatus and are uncorrected. ${ }^{~} \mathrm{H}$ NMR and ${ }^{13} \mathrm{C}$ NMR spectra were recorded on a JEOL $J$ NM/ EX-400 using $\mathrm{CDCl}_{3}$ as the solvent. All chemical shifts ( $\delta$ ) are quoted in ppm downfield from TMS and coupling constants $(S)$ are given in $\mathrm{Hz}$. Mass spectra were measured on a Agilent $1100 \mathrm{LC} / \mathrm{MSD}$ (API-ES) mass spectrometer.

2-(+-Hydroxyphenyl)-4-quinolinecarboxylic acid (1). A solution of aniline $(9.30 \mathrm{~g} .0 .10 \mathrm{~mol})$ in EtOH $(30 \mathrm{~mL})$ was added to a solution of py ruvic acid $(13.19 \mathrm{~g} .0 .15 \mathrm{~mol})$ and 4-hyddroxy benzaldehyde $(12.20 \mathrm{~g} .0 .10 \mathrm{~mol})$ in $\mathrm{EtOH}(80$ $\mathrm{mL}$ ). and the mixture was heated under reflux for $3 \mathrm{~h}$ and allowed to cool overnight. The resulting solid was collected by filtration washed sequentially with cold $\mathrm{EtOH}$ and benzene. and dried to give $1(13.51 \mathrm{~g} .51 \%)$ as a light yellow powder. $\mathrm{mp}=>300^{\circ} \mathrm{C}$. ${ }^{1} \mathrm{H}$ NMR $\left(400 \mathrm{MHz}\right.$, DMSO- $\left.d_{6}\right): \delta 9.76$ (br s. $1 \mathrm{H}), 8.71$ (d. $1 \mathrm{H}, J=8.30), 8.37$ (s. $1 \mathrm{H}) .8 .13$ (d. $2 \mathrm{H}, J=$ $8.79) .8 .09$ (d. $1 \mathrm{H}, J=8.30), 7.76(\mathrm{dd}, \mathrm{lH} . J=7.8 \mathrm{l}, 7.32)$, 7.65 (dd. $\mathrm{lH}, J=8.30,6.84) .6 .95$ (d. $2 \mathrm{H}, J=8.79) .{ }^{1.3} \mathrm{C} \mathrm{NMR}$ (100 MHz, DMSO- $d_{6}$ ): $\delta$ 167.56, 159.24. 155.65. 148.46. 136.61. 129.42. 129.31, 128.83, 128.42. 126.55. 125.29. 123.08. 118.72. 115.58, 115.53. MS: $m z(\%)=265(27$. M) $20+(100)$.

2-(4-Propoxyphenyl)-4-quinolinecarboxylic acid (2). Yield: $3.90 \mathrm{~g}(6.5 \%)$ as a light yellow powder. $\mathrm{mp}=194-198$ ${ }^{\circ} \mathrm{C} .{ }^{~} \mathrm{H}$ NMR $\left(400 \mathrm{MHz}, \mathrm{DMSO}-d_{6}\right): \delta 8.80$ (d. $1 \mathrm{H} . J=$ $8.30) .8 .41(\mathrm{~s}, 1 \mathrm{H}), 8.18(\mathrm{~d}, 2 \mathrm{H}, J=8.79) .8 .1+(\mathrm{d}, 1 \mathrm{H} . J=$ 8.30 ). 7.74 (t. $1 \mathrm{H} . J=7.32$ ). 7.57 (t. $1 \mathrm{H} . J=7.32$ ), $7.04(\mathrm{~d} .2 \mathrm{H}$, $J=8.79), 4.0 \mathrm{l}(\mathrm{dd}, 2 \mathrm{H}, J=6.84 .0 .49), 1.85(\mathrm{~m}, 2 \mathrm{H}) .1 .07(\mathrm{t}$. $3 \mathrm{H} . J=7.32) .{ }^{13} \mathrm{C}$ NMR (100 MHz, DMSO- $\left.d_{6}\right): \delta 167.89$, 160.17. 155.63. 148.62, 136.29, 130.50. 129.34. 129.19. $128.24,126.57,125.30,123.36,119.25,114.32 .69 .07 .22 .03$. 10.08. MS: $m z(\%)=307\left(18 . \mathrm{M}^{\prime}\right) .262(68), 204(100), 128(35)$.

N4-(1-Benzyl-4-piperidyl)-2-(4-hydroxyphenyl)-4-quinolinecarboxamide (3). To a solution of EDCI $(0.43 \mathrm{gg} .2 .26$ mmol) in dry DMF (5 mL) was added quinolinecarboxylic acid $(0.20 \mathrm{~g} .0 .75 \mathrm{mmol})$ in dry DMF $(5 \mathrm{~mL})$. The solution was stirred at room temperature for $30 \mathrm{~min}$. To this mixture were added 4-amino-l-benzylpiperidine ( $0.77 \mathrm{~mL} .3 .77$ mmol) in dry DMF ( $2 \mathrm{~mL}$ ) and DMAP (cat.). stirred at room temperature for $16 \mathrm{~h}$. The reaction was quenched with $\mathrm{H}_{2} \mathrm{O}$ $(100 \mathrm{~mL})$, extracted with EtOAc $(20 \mathrm{~mL} \times 2)$. The extracts were washed with $\mathrm{H}_{2} \mathrm{O}$ and brine, dried $\left(\mathrm{Na}_{2} \mathrm{SO}_{1}\right)$. and evaporated. The residue was purified by column chromatography ( $10 \% \mathrm{MeOH}$ : EtOAc) to give $3(0.25 \mathrm{~g} .76 \%$ ) as a white powder $\mathrm{mp}=222-226{ }^{\circ} \mathrm{C}$. ${ }^{1} \mathrm{H}$ NMR $(400 \mathrm{MHz}$. $\left.\mathrm{CDCl}_{3}\right): \delta 8.16(\mathrm{~d} .1 \mathrm{H}, J=8.79), 8.13(\mathrm{~d} . \mathrm{lH} . J=8.79) .8 .08$ (d. $2 \mathrm{H}, J=8.79), 7.83(\mathrm{~s}, 1 \mathrm{H}) .7 .74(\mathrm{t}, 1 \mathrm{H}, J=7.32) .7 .54$ (t. IH. $J=7.32), 7.27(\mathrm{~m} .5 \mathrm{H}), 6.98(\mathrm{~d} .2 \mathrm{H} . J=8.79) .5 .93(\mathrm{~d}$. 1H. $J=7.6+\mathrm{NH}), 4.13(\mathrm{~m} . \mathrm{lH}) .3 .55(\mathrm{~s}, \mathrm{lH}) .2 .9 \mathrm{l}(\mathrm{m} .2 \mathrm{H})$. 2.25 (m. $2 \mathrm{H}) .2 .15$ (m. $2 \mathrm{H}) .1 .65(\mathrm{~m} .2 \mathrm{H}) .{ }^{13} \mathrm{C} \mathrm{NMR}(100$ $\left.\mathrm{MHz}, \mathrm{CDCl}_{3}\right): \delta 169.08,164.83,157.34 .150 .57,144.70$. 140.03. 134.24. 130.31, 129.78, 129.13. 128.30. 128.15 . 126.94. 126.76. 125.53, 123.41, 115.86. 113.99. 113.87. $62.96,52.26,+7.53 .32 .20 .22 .52$. MS: $m z(\%)=437(28$. M) $.+20(31), 218(52), 20+(100) .17+(+4) .128(+0)$.

N4-(1-Benzyl-4-piperidyl)-2-(4-pmpoxyphenyl)-4-quinolincearboxamide (4). Yield: $0.26 \mathrm{~g}(70 \%)$ as a white powder. $\mathrm{mp}=162-164^{\circ} \mathrm{C}$. 'H NMR $\left(400 \mathrm{MHz} . \mathrm{CDCl}_{3}\right): \delta 8.06$ (d. IH. $J=8.30$ ). 7.99 (d. 2 H. $J=8.79$ ). $7.93($ d. $1 \mathrm{H} . J=8.30$ ). 7.65 (t. $1 \mathrm{H}, J=7.32$ ). $7.6 \mathrm{l}$ (s. $1 \mathrm{H}$ ). 7.39 (t. $1 \mathrm{H}, J=7.32$ ). $7.33(\mathrm{~m}, \mathrm{HH}) .7 .28(\mathrm{~m} . \mathrm{lH}) .6 .97(\mathrm{~d} .2 \mathrm{H}, J=8.79) .6 .+4(\mathrm{~d}$. IH. $J=7.81, \mathrm{NH}) .4 .07(\mathrm{~m} .1 \mathrm{H}) .3 .97$ (t. $2 \mathrm{H} . J=6.84) .3 .52$ (s. $2 \mathrm{H}) .2 .89$ (m. $2 \mathrm{H}) .2 .18(\mathrm{~m} .2 \mathrm{H}) .2 .10(\mathrm{~m} .2 \mathrm{H}) .1 .85(\mathrm{~m}$. $2 \mathrm{H}) .1 .66(\mathrm{~m}, 2 \mathrm{H}) .1 .07$ (t. $3 \mathrm{H}, J=7.32) .{ }^{13} \mathrm{C}$ NMR $(100$ $\left.\mathrm{MHz}, \mathrm{CDCl}_{3}\right): \delta 167.04,160.63,156.14 .148 .37,1+2.76$. $138.33,130.84 .129 .91,129.57,129.02$. 128.75, 128.22. 127.04. 126.69. 124.73. 122.81, 115.78, 11+.69.69.57.62.96, 52.26. 47.53,32.20.22.52, 10.50. MS: $m z(\%)=479\left(39, \mathrm{M}^{\prime}\right),+36$ (21). $420(+3) .218(63), 204(100), 17+(40), 128(38)$.

N4-(1-Benzy]-4-piperidyl)-2-[4-(3-aminopmpoxy)phenyl]4-quinolinecarboxamide hydmchloride (6). To a solution 
of $1 \mathrm{M} \mathrm{NaOH}(20.10 \mathrm{~mL}, 20.10 \mathrm{mmol})$ in tert-butyl alcohol $(6 \mathrm{~mL})$ was added 3-bromopropy lamine lyydrobromide (2.00) g. $9.1+$ munol) and di-tert-butylcarbonate $(2.19$ g. 10.05 munol), the reaction mixture was stirred for 12 h at room temperature. The reaction mixture was washed with 0.1 t: $\mathrm{HCl}$ and $5 \% \mathrm{NaHCO}_{3}$, Brine. The organic layer was dried over $\mathrm{Na}_{2} \mathrm{SO}_{4}$ and concentrated in vacho to give $\mathrm{N}$-Boc-3bromopropy lamine $(1.40 \mathrm{~g} .64 \%)$ as a oil.

A solution of 1 -Boc-3-bromopropylamine $(0.14 \mathrm{~g} .0 .32$ munol) in $\mathrm{CH}_{3} \mathrm{CN}$ ( $\left.10 \mathrm{~mL}\right)$ was added to a mixture of $3(0.14$ g. $0.32 \mathrm{mmol}$ ) and $\mathrm{K}_{2} \mathrm{CO}_{3}(0.1+$ g. $0.96 \mathrm{mmol})$ in $\mathrm{CH}_{3} \mathrm{CN}$ $(20 \mathrm{~mL})$. and the mixture was heated under reflux for $12 \mathrm{~h}$. The inorganic material was filtered off and the solvent was exaporated in vacuo. The crude was extracted with EtOAc $(20 \mathrm{~mL} \times 2)$. washed with $\mathrm{H}_{2} \mathrm{O}(100 \mathrm{~mL})$ and Brine. The extract was dried over $\mathrm{Na}_{2} \mathrm{SO}_{4}$ and concentrated in vacuo to give $5(0.17 \mathrm{~g} .89 \%)$ as a white powder.

A solution of $5(0.1+$ g. $0.2+\mathrm{mmol})$ in dry THF $(10 \mathrm{~mL})$ was cooled to $-20^{\circ} \mathrm{C}$ and stirred for $30 \mathrm{~min}$. Etherate $\mathrm{HCl}$ (lih) was added dropwise to the reaction mixture under $\mathrm{pH}=1$. The precipitate was collected by suction filtration, washed with ether $(5 \mathrm{~mL} \times 2)$ and dried in desiccator to give $6(0.12 \mathrm{~g} .96 \%)$ as a light yellow solid. $\mathrm{mp}=174-179^{\circ} \mathrm{C} .{ }^{1} \mathrm{H}$ NMR ( $400 \mathrm{MHz}$, DMSO- $(6): \delta 8.44$ (d. $1 \mathrm{H} . J=8.30) .8 .41$ (s. $1 \mathrm{H}) .8 .36(\mathrm{~d} .1 \mathrm{H} . J=8.30), 8.26(\mathrm{~d} .2 \mathrm{H}, J=8.79), 8.15(\mathrm{t}$. $1 \mathrm{H} . J=7.81) .7 .93(\mathrm{t} . \mathrm{lH} . J=7.81) .7 .60(\mathrm{~m} .2 \mathrm{H}) .7 .51(\mathrm{~m}$. $3 \mathrm{H}) .7 .32(\mathrm{~d} .2 \mathrm{H} . J=8.79), 4.3+(\mathrm{ml} . \mathrm{lH}), 4.30(\mathrm{~m}, 2 \mathrm{H}) .3 .6 \mathrm{l}$ (m. $2 \mathrm{H}) .3 .30$ (s. $2 \mathrm{H}) .3 .28$ (m. $2 \mathrm{H}) .3 .2 \mathrm{l}$ (m. $2 \mathrm{H}) .2 .40$ (m. $2 \mathrm{H}) .2 .24$ (m. $2 \mathrm{H}), 2.07$ (m. $2 \mathrm{H}){ }^{1.3} \mathrm{C}$ NMR $(100 \mathrm{MHz}$. DMSO-(t): $\delta$ 166.39, $16+65.156 .45 .151 .+2.1+0.64$. 136.05. 132.97. 132.76, 132.5t. 132.+1. 131.30, 131.23, 131.03. 130.50. 127.67, 125.29. 124.60. 122.15, 121.33, 120.70. 117.02, 66.93. 61.59, 52.70, +6.81, 38.39, 29.82 . 28.24. 24.22. MS: $m z(\%)=531(69 . \mathrm{M}) .420(5+), 218$ (+1). $20+(100) .174(36) .128(21)$.

N4-(1-Benzyl-4-piperidyl)-2-(4-isobutoxyphenyl)-4-quinolinecarboxamide (7). A solution of l-bromo-2-methy propane $(0.07 \mathrm{~g} .0 .50 \mathrm{mmol})$ in $\mathrm{CH}_{3} \mathrm{CN}(10 \mathrm{~mL})$ was added to a mixture of $3(0.20 \mathrm{~g} .0 .46 \mathrm{mmol})$ and $\mathrm{K}_{2} \mathrm{CO}_{3}(0.19 \mathrm{~g}$. $1.37 \mathrm{mmol})$ in $\mathrm{CH}_{3} \mathrm{CN}(20 \mathrm{~mL})$, and the mixture was heated under reflux for $12 \mathrm{~h}$. The inorganic material was filtered off and the solvent was exaporated in vacto. The cnide was extracted with EtOAc $(20 \mathrm{~mL} \times 2)$, washed with $\mathrm{H}_{2} \mathrm{O}(100)$ $\mathrm{mL}$ ) and Brine. The extract was dried over $\mathrm{Na}_{2} \mathrm{SO}_{4}$ and concentrated in vacto to give $7(0.17 \mathrm{~g} .77 \%$ ) as a white powder. $\mathrm{mp}=112-114^{\circ} \mathrm{C}$. 'H NMR $\left(400 \mathrm{MHz}, \mathrm{CDCl}_{3}\right): \delta 8.06(\mathrm{~d}$. $1 \mathrm{H}, J=8.30$ ). 7.99 (d. $2 \mathrm{H}, J=8.79$ ). 7.93 (d. $1 \mathrm{H} . J=8.30$ ), 7.65 (t. $1 \mathrm{H} . J=7.32$ ) 7.62 (s. $1 \mathrm{H}$ ). 7.39 (t. $1 \mathrm{H} . J=7.32$ ). $7.33(\mathrm{~m} .4 \mathrm{H}) .7 .28(\mathrm{~m} . \mathrm{HH}) .6 .97$ (d. $2 \mathrm{H} . J=8.79), 6.48(\mathrm{~d}$. $1 \mathrm{H} . J=8.30 \mathrm{NH}), 4.09(\mathrm{~m} . \mathrm{lH}) .3 .77(\mathrm{~d} .2 \mathrm{H} . J=6.35) .3 .52$ $(\mathrm{s} .3 \mathrm{H}) .2 .89(\mathrm{~m} .2 \mathrm{H}) .2 .23(\mathrm{~m} .3 \mathrm{H}) .2 .10(\mathrm{~m} .2 \mathrm{H}) .1 .68(\mathrm{~m}$. $2 \mathrm{H}) .1 .06$ (d. $6 \mathrm{H} . J=6.35) .{ }^{13} \mathrm{C}$ NMR $\left(100 \mathrm{MHz} . \mathrm{CDCl}_{3}\right): \delta$ 167.05. 160.76. 156.13, 148.35. 142.75. 138.31, 130.79 . 129.89. 129.55. 129.02, 128.72. 128.20. $127.03,126.66$. $12+.73 .122 .80,115.78,114.70,62.94,60.3+.52 .25,47.52$, $32.16,28.24 .19 .2+.1+.13 . \mathrm{MS}: m z(\%)=493\left(+7 . \mathrm{M}^{\prime}\right)+20$
(50). $218(38) .204(100) .174(40), 128(29)$.

$N 4$-(1-Benzyl-t-piperidyl)-2-[t-(2-propy Inyloxy)phenyl]t-cuinolinecarboxamide (8). Yield: $0.80 \mathrm{~g}(36 \%)$ as a white powder. $\mathrm{mp}=104-106{ }^{\circ} \mathrm{C} .{ }^{1} \mathrm{H}$ NMR $(400 \mathrm{MHz}$. $\mathrm{CD}_{3} \mathrm{OD}$ ): $\delta 8.06$ (d. $1 \mathrm{H} . J=8.79$ ). 8.02 (d. $2 \mathrm{H} . J=8.30$ ). 7.86 (s. $1 \mathrm{H}), 7.68$ (t. $1 \mathrm{H} . J=7.32$ ), 7.50 (t. $1 \mathrm{H} . J=7.32$ ), $7.18(\mathrm{~m},+\mathrm{H}), 7.11(\mathrm{~m} . \mathrm{lH}), 7.03(\mathrm{~d} .2 \mathrm{H} . J=8.79), 4.73(\mathrm{~s}$. $2 \mathrm{H}) .3 .98(\mathrm{~m} . \mathrm{lH}) .3 .2 \mathrm{l}(\mathrm{s}, 2 \mathrm{H}) .2 .90(\mathrm{~s}, \mathrm{lH}) .2 .84(\mathrm{~m} .2 \mathrm{H})$. 2.03 (m. 2H). 1.65 (m. 4H) ${ }^{1.3} \mathrm{C} \mathrm{NMR}\left(100 \mathrm{MHz} . \mathrm{CD}_{3} \mathrm{OD}\right)$ : $\delta 169.53,160.77 .158 .07 .149 .63,143.05,133.25 .131 .47$. 130.46. 130.29, 130.22, 130.10, 129.28, 128.15. 128.07. 127.62. 126.02. 124.58, 117.87, 116.33.79.55.77.08,60.51. $56.73,46.98,4+23,40.41,29.47,20.85$. MS: $m z(\%)=475$ (16, M). $420(63) .218(44), 204$ (100). $174(48), 128(36)$.

Acknowledgement. This work was supported by a grant from the International Mobile Telecommunication 2000 R\&D Project (01-PJ11-PG9-0lBT07-0002), Ministry of Information \& Communication. Republic of Korea.

\section{References}

1. Dickinson. R. B.: McManaway. M. E.: Lippman. M. E. Science $1986,232.1540$

2. Tsai, M. I.: OMallex: B. W. Ammi. Rev Biochem 1994. 63.451.

3. Sun, I.: Mevers. M. J.: Fink, B. F.: Rajendran, R.: Katenellenbogen. J. A.: Kalzenellenbogen. B. S. Endocrinoliggv 1999. 1t0. 800.

4. Wiese. T. E.: Polin. L. A.: Palomino. E.: Brooks. S. C. J. Ifed (hem 1997. t0. 3659)

5. I.i, J. I. I Iou. X.; Bentel, J.: Yazloritskava. F. M.: I.i. S. A. Cancinogenesis 1998. 19,471.

6. I.ippman, M. F.: Bolan, G. Sature 1975, 256. 592

7. Jordan. V. C. Fur.J. Cancer 1976. 12. 419

8. Jordan. V. C.: Morrow. M. Br. Hed J. 1999. 319.331

9. Munsler. P. N.: Buzdar. A.: Dhingra. K.: Enas. N.: Ni. L.: Major. M.: Melemed, A.: Scidman. A.: Booser, D.: Theriault, R.: Norton. I... Iludis. C. J. Chin. Oncol. 2001. 19.2002.

10. I.abrie, F.: I abric, C.: Belanger. A.; Simard. I.: Gauthicr. S.: I uuThe. V: Merand. Y.: Giguere. V.: Candas. B.: Luo. S.: Martel. C.: Singh. S. M.: Fournier. M.: Coglet. A.: Richard. V: Charbonneau. R.: Charpenet. G.: Tremblay. A.: Tremblay: G.: Cusan. L.: Veilleux. R. J. Sthord Biochem. Hol Biol. 1999. 69. 51

11. Ke. II. 7.: Qi, II.: Chidsey-Frink, K. I..: Crantord, D. T.: Thompson, D. D. J. Bone Winer Res. 2001. 16. 765.

12. Doebner. O. Be?. 1883. 16.2357.

13. Atwell. G. J.: Baguley. B. C.: Denny. W. A. f. hed ("th'm. 1989. 32.396

14. (a) Dhaon. M. K.: Olsen. R. K.: Ramasams: K. J. Org. Chem. 1982, 47, 1962. (b) Desai. M. C.: Stcphens Stramiello. I.. M. Fetratredron Lett. 1993, 3f, 7685. (c) Akaji. K.: Kurivama. N.: Kiso. Y. Tetruhedryt Ieft 1994. 35. 3315. (d) Norman. B. H: Hemscheidt. T.: Schultz. R. M.: Andis. S. L. J. Org. Chem. 1998. 63. 5288 .

15. Saari, W. S.: Schwering. I. Г... I Ale. P. A.: Smith. S. I.: Fingcllardt,

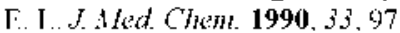

16. (a) Fleteher, S. R.; Burkamp, F.: Blurton, P.: Cheng. S. K. F.: Clarkson. R.: O'Connor. D.: Spinks. D.: Tudge. M.: ran Niel. M. B.: Patel. S.: Chapman. K.: Marwood. R.: Shepheard. S.: Bentley. G.: Cook. G. l:: Bristonm. L. I.: Castro. I. L.: Hutson. P. H.: MacI ed. ^. M. J. Hed. Chem 2002, 45, 492. (b) Salvatorc. R. N.: Yoon, C. It.: Jung. K. W. Tetrohedron 2001 . 57. 7785

17. Um, S. I.; Kim, F. I.: Uwang. F. S.: Kim, S. I.; Namkoong. S. F.: l'ark. I. S. Int. J. Cance'r 2000. \$5. 416 